CERN-TH/99-234

hep-th/9908014

\title{
TOWARDS THE CLASSIFICATION OF CONFORMAL FIELD THEORIES IN ARBITRARY EVEN DIMENSION
}

\author{
Damiano Anselmi \\ CERN, Theory Group, CH-1211, Geneva 23, Switzerland
}

\begin{abstract}
I identify the class of even-dimensional conformal field theories that is most similar to two-dimensional conformal field theory. In this class the formula, elaborated recently, for the irreversibility of the renormalization-group flow applies also to massive flows. This implies a prediction for the ratio between the coefficient of the Euler density in the trace anomaly (charge a) and the stress-tensor two-point function (charge $c$ ). More precisely, the trace anomaly in external gravity is quadratic in the Ricci tensor and the Ricci scalar and contains a unique central charge. I check the prediction in detail in four, six and eight dimensions, and then in arbitrary even dimension.
\end{abstract}

Pacs: 11.25.H; 11.10.Gh; 11.15.Bt; 11.40.Ex; 04.62.+v 
Four-dimensional conformal field theories have two central charges, $c$ and $a$, defined by the trace anomaly in a gravitational background. The charge $c$ multiplies the conformal invariant $W_{\mu \nu \rho \sigma}^{2}$ (square of the Weyl tensor) and is the coefficient of the two-point function of the stress tensor. The quantity a multiplies the Euler density $\mathrm{G}_{4}=\varepsilon_{\mu \nu \rho \sigma} \varepsilon^{\alpha \beta \gamma \delta} R_{\alpha \beta}^{\mu \nu} R_{\gamma \delta}^{\rho \sigma}$. A third term, $\square R$, is multiplied by a coefficient $a^{\prime}$ :

$$
\Theta=\frac{1}{(4 \pi)^{2}}\left[-c W^{2}+\frac{a}{4} \mathrm{G}_{4}-\frac{2}{3} a^{\prime} \square R\right]
$$

where $c=\frac{1}{120}\left(N_{s}+6 N_{f}+12 N_{v}\right), a=\frac{1}{360}\left(N_{s}+11 N_{f}+62 N_{v}\right)$ for free field theories of $N_{s, f, v}$ real scalars, Dirac fermions and vectors, respectively.

In higher, even dimension $n$ the trace anomaly contains more terms, which can however be grouped into the same three classes as in four dimensions. Several terms are exactly invariant under conformal transformations and are not total derivatives. They generalize $W^{2}$. The constants in front of these terms will be denoted collectively by $c$. One of such central charges, in particular, is related to the stress-tensor two-point function. It multiplies an invariant of the form $W_{\mu \nu \rho \sigma} \square^{n / 2-2} W^{\mu \nu \rho \sigma}+\mathcal{O}\left(W^{3}\right)$. The central charge $a$ is always unique and multiplies the Euler density $\mathrm{G}_{n}$, which is not conformally invariant, but a non-trivial total derivative. Finally, the constants in front of the trivial total derivatives, which generalize $\square R$, will be collectively denoted by $a^{\prime}$.

Only in two dimensions [1] has the trace anomaly a unique term, the Ricci scalar $R$. In some sense, we can say that " $c=a=a^{\prime \prime}$ " there. It is natural to expect that there exists a special class of higher-dimensional conformal field theories that is most similar to two-dimensional conformal field theory. This class will have to be identified by a universal relationship between the central charges $c, a$ and $a^{\prime}$.

The main purpose of this paper is to identify this class of conformal theories, collecting present knowledge and offering further evidence in favour of the statement. I first use the sum rule of refs. [2, 3] for the irreversibility of the renormalization-group flow to derive a quantitative prediction from this idea, namely the ratio between the coefficient $a_{n}$ of the Euler density $\mathrm{G}_{n}$ and the coefficient $c_{n}$ of the invariant $W_{\mu \nu \rho \sigma} \square^{n / 2-2} W^{\mu \nu \rho \sigma}+\mathcal{O}\left(W^{3}\right)$ (or, which is the same, the constant in front of the stress-tensor two-point function). Secondly, I argue that the conformal field theories of our special class are also those whose trace anomaly in external gravity is quadratic in the Ricci tensor and Ricci scalar. This property relates unambigously the central charges $c$ to the unique central charge $a$ and, in particular, should agree with the ratio $c_{n} / a_{n}$ found using the irreversibility of the RG flow.

I then proceed to check the prediction. This is first done in detail in four, six and eight dimensions and then extended to the general case. The results are also a very non-trivial test of the ideas of refs. [2, 3] about the irreversibility of the RG flow.

I recall that in [4, 5] it was shown that in four dimensions there is a "closed limit", in which the stress-tensor operator product expansion (OPE) closes with a finite number of operators up to the regular terms. The idea of this limit was suggested by a powerful theorem, due to Ferrara, Gatto and Grillo [6] and to Nachtmann [7], on the spectrum of anomalous dimensions 
of the higher-spin currents generated by the OPE, which follows from very general principles (unitarity) and is therefore expected to hold in arbitrary dimension.

When $c=a$ [4], OPE closure is achieved in a way that is reminiscent of two-dimensional conformal field theory, with the stress tensor and the central extension. Instead, when $c \neq a$ the algebraic structure is enlarged and contains spin- 1 and spin- 0 operators, yet in finite number. Therefore, the subclass of theories we are interested in is identified, in four dimensions, by the equality of $c$ and $a$ and the closed limit. Secondly, it is well known that $\Theta$ vanishes on Ricci-flat metrics when $c=a$ in four dimensions. A closer inspection of (11) shows that actually $\Theta$ is quadratic in the Ricci tensor and the Ricci scalar. We are led to conjecture that the subclass of " $c=a$ "-theories in arbitrary even dimension are those that have a trace anomaly quadratic in the Ricci tensor and the Ricci scalar.

Summarizing, in arbitrary even dimension greater than 2 we can distinguish the following important subclasses of conformal field theories:

i) The "closed" theories, when the quantum conformal algebra, i.e. the algebra generated by the singular terms of the stress-tensor OPE, closes with a finite number of operators. They can have $c=a$ [4], but also $c \neq a$ [5].

ii) The $c=a$-theories, whose trace anomaly is quadratic in the Ricci tensor and the Ricci scalar. They can be either closed or open.

iii) The closed $c=a$-theories, which exhibit the highest degree of similarity with twodimensional conformal field theory.

While the equality $c=a$ is a restriction on the set of conformal field theories, the equality of $a$ and $a^{\prime}$ is not. In refs. 2, 3] the equality $a=a^{\prime}$ was studied in arbitrary even dimension $n$, leading to the sum rule

$$
a_{n}^{\mathrm{UV}}-a_{n}^{\mathrm{IR}}=\frac{1}{2^{\frac{3 n}{2}-1} n n !} \int \mathrm{d}^{n} x|x|^{n}\langle\Theta(x) \Theta(0)\rangle,
$$

expressing the total renormalization-group (RG) flow of the central charge $a_{n}$, induced by the running of dimensionless couplings. This formula was checked to the fourth-loop order included in the most general renormalizable theory in four [2] and six [3] dimensions. No restriction on the central charges $c$ and $a$ is required here. The charge $a_{n}$ is normalized so that the trace anomaly reads

$$
\Theta=a_{n} \mathrm{G}_{n}=a_{n}(-1)^{\frac{n}{2}} \varepsilon_{\mu_{1} \nu_{1} \cdots \mu_{\frac{n}{2}} \nu_{\frac{n}{2}}} \varepsilon^{\alpha_{1} \beta_{1} \cdots \alpha_{\frac{n}{2}} \beta_{\frac{n}{2}}} \prod_{i=1}^{\frac{n}{2}} R_{\alpha_{i} \beta_{i}}^{\mu_{i} \nu_{i}}
$$

plus conformal invariants and trivial total derivatives.

As it was explained in the introduction of [2], the arguments of [2, 3] do not necessarily apply to flows generated by super-rinormalizable couplings and mass terms. (In general, the effect of masses can be included straightforwardly [8].) The sum rule (2) measures the effect of the dynamical RG scale $\mu$ in lowering the amount of massless degrees of freedom of the theory along the RG flow.

The basic reason why massive flows behave differently is that in a finite theory Duff's identification [9] $a^{\prime}=c$ is consistent (but not unique), while along a RG flow the only consistent 
identification is $a^{\prime}=a$, as shown in [2]. Divergences are crucial in discriminating between the two cases. A flow induced by divergences cannot, in general, be assimilated to a flow induced by explicit ("classical") scales.

Repeating the arguments of [2, 3] in two dimensions, we would come to the same conclusion as in higher dimensions: that the sum rule (2) works for RG flows and not necessarily for massive ones. The point is, nevertheless, that the two-dimensional version of (2), due to Cardy [10], is universal; in particular, it does work for massive flows. It is therefore compulsory to understand in what cases the domain of validity of our sum rule (2) is similarly enhanced in higher dimensions. This property identifies the special class of theories we are looking for.

The arguments and explicit checks that we now present show that this enhancement takes place in the subclass of theories with $c=a$ (classes $i i$ and $i i i$ above), because of the higher similarity with the two-dimensional theories.

The two relevant terms of the trace anomaly are

$$
\Theta=a_{n} \mathrm{G}_{n}-\frac{c_{n}(n-2)\left(\frac{n}{2}\right) !}{4(4 \pi)^{\frac{n}{2}}(n-3)(n+1) !} W \square^{\frac{n}{2}-2} W+\cdots,
$$

where

$$
c_{n}=N_{s}+2^{\frac{n}{2}-1}(n-1) N_{f}+\frac{n !}{2\left[\left(\frac{n}{2}-1\right) !\right]^{2}} N_{v}
$$

is the value of the central charge $c$ for free fields, and in arbitrary dimension $n . N_{v}$ denotes the number of $(n / 2-1)$-forms. This calculation is done in ref. [11], section 9 , starting from the stress-tensor two-point function.

Massive flows have been considered, among other things, by Cappelli et al. in [12]. An explicit computation for free massive scalar fields and fermions gives [12]

$$
\int \mathrm{d}^{n} x|x|^{n}\langle\Theta(x) \Theta(0)\rangle=\frac{c_{n}\left(\frac{n}{2}\right) !}{\pi^{\frac{n}{2}}(n+1)} .
$$

Repeating the computation for massive vectors, or $(n / 2-1)$-forms, is problematic in the UV. However, the relative coefficient between the scalar and fermion contributions is sufficient to show that the result is proportional to $c_{n}$ and not $a_{n}$.

Our prediction is that in the special $c=a$-theories the sum rule (2) should reproduce (3) for massive flows, which means

$$
c_{n}=a_{n} \frac{2^{\frac{n}{2}-1}(4 \pi)^{\frac{n}{2}} n(n+1) !}{\left(\frac{n}{2}\right) !} .
$$

The trace anomaly therefore has the form

$$
\Theta=a_{n}\left(\mathrm{G}_{n}-\frac{2^{\frac{n}{2}-3} n(n-2)}{n-3} W \square^{n / 2-2} W\right)+\cdots
$$

Formula (四) is the generalized version of the relation $c=a$. It is uniquely implied by the requirement that $\Theta$ be quadratic in the Ricci tensor and Ricci curvature. This condition fixes all 
the central charges of type $c$ in terms of $a_{n}$, not only the constant $c_{n}$ in front of the stress-tensor two-point function. These further relationships are not important for our purposes.

In four dimensions the combination between the parenthesis in (5) is indeed quadratic in the Ricci tensor:

$$
\frac{\mathrm{G}_{4}}{4}-W^{2}=-2 R_{\mu \nu}^{2}+\frac{2}{3} R^{2} .
$$

I stress that this is a non-trivial check of the prediction that formula (2) correctly describes massive flows when $c=a$.

In higher dimensions the check is less straightforward, owing to the high number of invariants. Using the results of Bonora et al. from [13] (see also [14]), where the terms occurring in the trace anomaly were classified in six dimensions, we can perform a second non-trivial check of our prediction. The conformal invariants are three:

$$
\begin{aligned}
& I_{1}=W_{\mu \nu \rho \sigma} W^{\mu \alpha \beta \sigma} W_{\alpha \beta}^{\nu}{ }^{\rho}, \quad I_{2}=W_{\mu \nu \rho \sigma} W^{\mu \nu \alpha \beta} W_{\alpha \beta}{ }^{\rho \sigma}, \\
& I_{3}=W_{\mu \alpha \beta \gamma}\left(\square \delta_{\nu}^{\mu}+4 R_{\nu}^{\mu}-\frac{6}{5} R \delta_{\nu}^{\mu}\right) W^{\nu \alpha \beta \gamma},
\end{aligned}
$$

and the general form of the trace anomaly is

$$
\Theta=a_{6} \mathrm{G}_{6}+\sum_{i=1}^{3} c^{(i)} I_{i}+\text { t.t.d., }
$$

where "t.t.d." means "trivial total derivatives" (as opposed to $\mathrm{G}_{6}$, which is a non-trivial total derivative). Our notation differs from the one of [13] in the signs of $R_{\mu \nu}$ and $R$. More importantly, the invariant $I_{3}$ differs from the invariant $M_{3}$ of [13] and other references [15], the latter containing a spurious contribution proportional to $\mathrm{G}_{6}$ (see also [3], section 3), as well as a linear combination of $I_{1}$ and $I_{2}$. Precisely, we find

$$
M_{3}=\frac{5}{12} \mathrm{G}_{6}+\frac{80}{3} I_{1}+\frac{40}{3} I_{2}-5 I_{3} .
$$

Finally, our $I_{3}$ differs from the expression of ref. [16], formula (19), by the addition of t.t.d.'s, which, however, can be consistently omitted for our purposes.

In [13] it is pointed out that there exists a simple combination of the four invariants $\mathrm{G}_{6}$ and $I_{1,2,3}$, which reads

$$
\begin{aligned}
\mathcal{J}_{6}= & R_{\mu \nu} \square R^{\mu \nu}-\frac{3}{10} R \square R-R R_{\mu \nu} R^{\mu \nu} \\
& -2 R_{\mu \nu} R_{\rho \sigma} R^{\mu \rho \sigma \nu}+\frac{3}{25} R^{3} \\
= & -\frac{1}{24} \mathrm{G}_{6}-4 I_{1}-I_{2}+\frac{1}{3} I_{3}+\text { t.t.d. }
\end{aligned}
$$

The BPB (Bonora-Pasti-Bregola) term $\mathcal{J}_{6}$ is precisely the combination we are looking for. A closer inspection of this expression shows that it is uniquely fixed by the requirement that it be quadratic in the Ricci tensor and Ricci curvature. On the other hand, the requirement that $\mathcal{J}_{6}$ 
just vanishes on Ricci-flat metrics is not sufficient to fix it uniquely, in particular it does not imply the relation " $c=a$ " that we need.

In conclusion, the $c=a$-theories have a unique central charge, multiplying the BPB invariant $\mathcal{J}_{6}$

$$
\Theta=-24 a_{6} \mathcal{J}_{6}, \quad c^{(1)}=96 a_{6}, \quad c^{(2)}=24 a_{6}, \quad c^{(3)}=-8 a_{6},
$$

so that $\Theta$ is of the predicted form (5):

$$
\Theta=a_{6}\left(\mathrm{G}_{6}-8 W \square W\right)+\cdots
$$

Our prediction is meaningful in arbitrary even dimension and can be checked using the recent work of Henningson and Skenderis [17], which contains, as I now discuss, an algorithm to generate precisely the invariants $\mathcal{J}_{n}$ 's that we need. It is easy to verify this in four and six dimensions. In six dimensions the result can be read from formula (30) of [17, taking into account that in [17] the BPB invariant $M_{3}$ is used. A more convenient decomposition of the anomaly into Euler density and conformal invariants is the last equality of (6), leading directly to (7). It is therefore natural to expect that the algorithm of [17] answers our question and constructs the invariants $\mathcal{J}_{n}$ 's. I now check agreement with formula (5) in arbitrary even dimension.

I begin with $n=8$. The relevant terms of $\mathcal{J}_{8}$ are

$$
\mathcal{J}_{8}=R_{\mu \nu} \square^{2} R^{\mu \nu}-\frac{2}{7} R \square^{2} R+\mathcal{O}\left(R^{3}\right)=\alpha_{8} \mathrm{G}_{8}+\text { c.i. }+ \text { t.t.d. }
$$

$\alpha_{8}$ being the unknown coefficient and "c.i." denoting conformal invariants. On a sphere, in particular, all terms but $\alpha_{8} \mathrm{G}_{8}$ vanish, so that $\alpha_{8}$ can be found by evaluating the integral of $\mathcal{J}_{8}$ :

$$
\int_{S^{8}} \sqrt{g} \mathcal{J}_{8} \mathrm{~d}^{8} x=768 \alpha_{8}(4 \pi)^{4}
$$

Using

$$
W \square^{2} W=\frac{10}{3}\left(R_{\mu \nu} \square^{2} R^{\mu \nu}-\frac{2}{7} R \square^{2} R\right)+\mathcal{O}\left(R^{3}\right)+\text { t.t.d. }
$$

our prediction (5) is $\alpha_{8}=-1 / 64$. Indeed, applying the method of [17] on a conformally-flat metric with $R_{\mu \nu}=\Lambda g_{\mu \nu}$, we get, after a non-trivial amount of work,

$$
\mathcal{J}_{8}=\alpha_{8} \mathrm{G}_{8}=-\frac{1440}{343} \Lambda^{4}
$$

which gives the desired value of $\alpha_{8}$.

The check can be generalized for arbitrary $n$. The invariant $\mathcal{J}_{n}$ is, up to an overall factor $\beta_{n}$, the coefficient of $\rho^{n / 2}$ in the expansion of $\sqrt{\operatorname{det} G}$, where

$$
G_{\mu \nu}=g_{\mu \nu}+\sum_{k=1}^{n / 2} \rho^{k} g_{\mu \nu}^{(k)}+\mathcal{O}\left(\rho^{n / 2} \ln \rho, \rho^{n / 2+1}, \cdots\right)
$$


and the $\rho$-dependence is fixed by the equations 17

$$
\begin{aligned}
\operatorname{tr}\left[G^{-1} G^{\prime \prime}\right]-\frac{1}{2} \operatorname{tr}\left[G^{-1} G^{\prime} G^{-1} G^{\prime}\right]= & 0, \\
2 \rho\left(G^{\prime \prime}-G^{\prime} G^{-1} G^{\prime}\right)= & \left(G-\rho G^{\prime}\right) \operatorname{tr}\left[G^{-1} G^{\prime}\right] \\
& +\operatorname{Ric}(G)+(n-2) G^{\prime}
\end{aligned}
$$

Precisely,

$$
\begin{aligned}
\left.\frac{1}{\left(\frac{n}{2}\right) !} \frac{\mathrm{d}^{\frac{n}{2}}}{\mathrm{~d} \rho^{\frac{n}{2}}} \frac{\sqrt{\operatorname{det} G}}{\sqrt{\operatorname{det} g}}\right|_{\rho=0}=\beta_{n} \mathcal{J}_{n} \\
\quad \beta_{n}\left(R_{\mu \nu} \square^{\frac{n}{2}-2} R^{\mu \nu}+\alpha_{n} \mathrm{G}_{n}+\text { rest }\right) .
\end{aligned}
$$

First, we consider metrics with $R_{\mu \nu}=\Lambda g_{\mu \nu}$. The form of the solution and the first equation of (8) read

$$
G_{\mu \nu}=u(\rho \Lambda) g_{\mu \nu}, \quad \frac{u^{\prime \prime}}{u}=\frac{1}{2}\left(\frac{u^{\prime}}{u}\right)^{2} .
$$

The second equation of (8) is used to fix the integration constants, with the result

$$
u(\rho \Lambda)=\left(1-\frac{\rho \Lambda}{4(n-1)}\right)^{2}, \quad \beta_{n} \mathcal{J}_{n} \rightarrow \frac{(-1)^{\frac{n}{2}} n ! \Lambda^{\frac{n}{2}}}{2^{n}(n-1)^{\frac{n}{2}}\left[\left(\frac{n}{2}\right) !\right]^{2}} .
$$

Then, we fix the normalization $\beta_{n}$ by looking for the term $R_{\mu \nu} \square^{\frac{n}{2}-2} R^{\mu \nu}$ (we can set the Ricci curvature $R$ to zero for simplicity). We write

$$
G_{\mu \nu}=g_{\mu \nu}+\frac{1}{\square} v(\rho \square) R_{\mu \nu}+R_{\mu \alpha} \frac{1}{\square^{2}} y(\rho \square) R_{\nu}^{\alpha}+O\left(R^{3}\right),
$$

with $v(0)=y(0)=y^{\prime}(0)=0$. We have

$$
\beta_{n}=\left.\frac{1}{2\left(\frac{n}{2}\right) !} \frac{\mathrm{d}^{\frac{n}{2}} x}{\mathrm{~d} t^{\frac{n}{2}}}\right|_{t=0}
$$

where $t=\rho \square$ and $x=y-v^{2} / 2$. Integrating $\mathcal{J}_{n}$ over a sphere, we can convert our prediction (5) to a prediction for $\beta_{n}$ or

$$
\left.\frac{\mathrm{d}^{\frac{n}{2}} x}{\mathrm{~d} t^{\frac{n}{2}}}\right|_{t=0}=-\frac{1}{2^{n-1} \Gamma\left(\frac{n}{2}\right)}
$$

Equations (8) relate $y$, and therefore $x$, to $v$ and imply that $v$ is a Bessel function of the second type:

$$
x^{\prime \prime}=-\frac{\left(v^{\prime}\right)^{2}}{2}, \quad 2 t v^{\prime \prime}-1+\frac{v}{2}-(n-2) v^{\prime}=0
$$

$\beta_{n}$ is a coefficient in the series expansion of the square of a Bessel function of the second type, and is not usually in the mathematical tables. Solving (10) recursively with the help of a calculator, we have checked agreement between (9) and (10) up to dimension 1000. 
Our picture and the quantitative agreement with prediction (5) explain, among other things, the physical meaning of the construction of ref. [17]. Furthermore, the mathematical properties of the invariant $\mathcal{J}_{n}$, and therefore the identification of $c$ and $a$ (in the subclasses of theories ii and $i i$ where it applies), are a nice counterpart of the notion of extended (pondered) Euler density introduced in [3], which explained the identification $a=a^{\prime}$. The results presented in this paper are a further check of the ideas of [2, 3] and of the picture offered there. These are, we believe, the first steps towards the classification of all conformal field theories.

The set of higher-dimensional quantum field theories, conformal or not, is not rich of physical models. Yet, one can consider higher-derivative theories, which, despite the issues about unitarity (see for example [18]), are useful toy-models for our purposes. Here higher-dimensional higher-derivative theories are meant as a convenient laboratory where the results of the present paper might be applied.

I thank A. Cappelli for reviving my interest for massive flows, the organizers of the $4^{\text {th }}$ Bologna Workshop on CFT and integrable models, D.Z. Freedman and N. Warner for stimulating conversations on the four-dimensional problem, M. Porrati for drawing my attention to the six-dimensional results of ref. [17], and finally L. Girardello and A. Zaffaroni.

\section{References}

[1] A.A. Belavin, A.M. Polyakov and A.B. Zamolodchikov, Infinite conformal symmetry in two-dimensional conformal field theory, Nucl. Phys. B 241 (1984) 333;

A.B. Zamolodchikov, "Irreversibility" of the flux of the renormalization group in a 2D field theory, JETP Lett. 43 (1986) 730.

[2] D. Anselmi, Anomalies, unitarity and quantum irreversibility, Ann. Phys. (NY) 276 (1999) 361 and hep-th/9903059.

[3] D. Anselmi, Quantum irreversibility in arbitrary dimension, hep-th/9905005, April 1999, to appear in Nucl. Phys. B.

[4] D. Anselmi, The N=4 quantum conformal algebra, Nucl. Phys. B 541 (1999) 369 and hep-th/9809192.

[5] D. Anselmi, Quantum conformal algebras and closed conformal field theory, Nucl. Phys. B 554 (1999) 415 and hep-th/9811149.

[6] S. Ferrara, R. Gatto and A. Grillo, Positivity constraints on anomalous dimensions, Phys. Rev. D 9 (1974) 3564.

[7] O. Nachtmann, Positivity constraints for anomalous dimensions, Nucl. Phys. B 63 (1973) 237.

[8] D. Anselmi, J. Erlich, D.Z. Freedman and A.A. Johansen, Positivity constraints on anomalies in supersymmetric theories, Phys. Rev. D57 (1998) 7570 and hep-th/9711035. 
[9] M. Duff, Observations on conformal anomalies, Nucl. Phys. B 125 (1977) 334.

N.D. Birrel and P.C.W. Davies, Quantum fields in curved space (Cambridge University Press, Cambridge, 1982), p. 180.

[10] J.L. Cardy, Central charge and the universal combinations of amplitdes in two-dimensional theories away from criticality, Phys. Rev. Lett. 60 (1988) 2709.

[11] D. Anselmi, Higher-spin current multiplets in operator-product expansions, hepth/9906167, June 1999.

[12] A. Cappelli, J.I. Latorre, X. Vilasis-Cardona, Renormalization-group patterns and ctheorem in more than two dimensions, Nucl. Phys. B 376 (1992) 510 and hep-th/9109041.

[13] L. Bonora, P. Pasti and M. Bregola, Weyl cocycles, Class. and Quantum Grav. 3 (1986) 635.

[14] S. Deser and A. Schwimmer, Phys. Lett. B 309 (1993) 279 and hep-th/9302047.

[15] D.R. Karakhayan, R.P. Manvelyan and R.L. Mkrtchyan, Trace anomalies and cocycles of Weyl and diffeomorphism groups, Mod. Phys. Lett. A 11 (1996) 409 and hep-th/9411068.

[16] T. Arakelyan, D. R. Karakhanyan, R. Manvelyan and R. Mkrtchyan, Trace anomalies and cocycles of Weyl group, Phys. Lett. B 353 (1995) 52.

[17] M. Henningson and K. Skenderis, The holographic Weyl anomaly, JHEP 9807 (1998) 023 and hep-th/9806087.

[18] I. Antoniadis, P.O. Mazur and E. Mottola, Physical states of the quantum conformal factor, Phys. Rev. D55 (1997) 4770 and hep-th/9509169. 